\title{
Gene expression profile of mesenchymal stromal cells after co-culturing with injured liver tissue
}

\author{
KYUNG-AH CHO ${ }^{1}$, SUN-YOUNG JU ${ }^{1}$, KYUNG-HA RYU ${ }^{2}$ and SO-YOUN WOO ${ }^{1}$ \\ Departments of ${ }^{1}$ Microbiology, and ${ }^{2}$ Pediatrics, School of Medicine, Ewha Womans University, Seoul, Korea
}

Received September 19, 2008; Accepted November 10, 2008

DOI: 10.3892/mmr_00000061

\begin{abstract}
Mesenchymal stromal cells (MSCs) are a potential cell source for the development of therapeutic products. Recent studies have shown that the transplantation of MSCs to damaged organs, including the heart, liver and kidneys, results in the restoration of the damaged tissues. However, the mechanisms underlying this regeneration process have yet to be clearly characterized. Consequently, in this study, we focused on the therapeutic potential of MSCs in injured liver tissue by evaluating the gene expression profiles of MSCs in the presence of injured liver and normal liver cells using a microarray chip containing 44,000 genes. In order to mimic the state of liver cell regeneration in vitro, we respectively cocultured MSCs with $\mathrm{CCl}_{4}$-injured liver cells and normal liver cells from C57BL/6 female mice. After $48 \mathrm{~h}$ of co-culturing, MSCs were collected and the RNA was extracted for microarray analysis. Under conditions of co-culture with normal liver cells, upregulated expression of CXCR6, CCR3, IL-2, IL-11, CD34, CD74, procollagen, FMS-like tyrosine kinase, neuregulin 4 , Wnt 2 and catenins was noted. Under conditions of co-culture with the $\mathrm{CCl}_{4}$-injured liver cells, expression of CXCL2, cytoglobin, erythropoietin, v-Erb, hypoxia-inducible factor 3 ( $\alpha$ subunit), retinoic acid receptor $\beta$ and Vav2 was upregulated. Our research provides information regarding the differential molecular mechanisms that regulate the properties of MSCs in the regeneration of injured liver tissue.
\end{abstract}

\section{Introduction}

Bone marrow (BM) contains heterogenous cells consisting of hematopoietic stem cells (HSCs) and stromal cells, which support the development of HSCs. Among such cells, multipotent stromal cells, or mesenchymal stromal cells (MSCs), perform a supportive role as stromal cells in BM, and also possess the potential to differentiate into a variety of cell types, including osteocytes, chondrocytes, adipocytes

Correspondence to: Dr So-Youn Woo, Department of Microbiology, School of Medicine, Ewha Womans University, 911-1 Mok-6-Dong, Yang-chun-gu, 158-710 Seoul, Korea

E-mail: soyounwoo@ewha.ac.kr

Key words: mesenchymal stromal cells, liver injury, microarray, gene expression and neuronal cells. Recently, the differentiation of MSCs into hepatic cell lineages has been reported. This implies that MSCs might potentially be employed as a source of cell-based therapy for the purpose of tissue regeneration $(1,2)$.

MSCs of this type residing in the BM can differentiate into hepatic cells, which express the liver-specific markers albumin, $\alpha$-fetoprotein and cytokeratin 18 as a result of stimulation with stem cell factor, epidermal growth factor and hepatocyte growth factor (3). MSCs can also be differentiated into hepatic lineage cells by co-culturing with liver tissues. In previous studies, MSCs were co-cultured with fetal liver cells (1) or liver cells, and were shown to differentiate into functional liver cells secreting albumin and urea after $48 \mathrm{~h}$ of co-culture (2).

We previously reported that $\mathrm{BM}$ cells ameliorate the pathologic conditions of $\mathrm{CCl}_{4}$-induced liver injury in mice (4). As MSCs derived from BM are one source of stem cells toward hepatic lineage, they principally differentiate into functional hepatocytes and may contribute to the regeneration of injured liver tissues. Therefore, we analyzed the gene expression profile of MSCs co-cultured with liver cells that mimicked the liver microenvironment in vitro. The gene expression profiles of MSCs were assessed after co-culturing with normal liver cells or with liver cells from $\mathrm{CCl}_{4}$-injected mice via a microarray technique.

\section{Materials and methods}

Mice. Six-week-old C57BL/6 mice were purchased from Koatec (Pyung-Taek, Korea). All mice were bred and housed under specific pathogen-free conditions. All procedures were approved by the Animal Care and Use Committee of the Ewha Womans University School of Medicine.

\section{Cell culture}

Isolation and culture of MSCs. Six- to eight-week-old C57BL/6 female mice were sacrificed by cervical dislocation and their limbs were removed. The BM was flushed from the medullary cavities of both the femurs and tibias with serumfree RPMI-1640 medium (Gibco BRL, Carlsbad, CA) using a 25-gauge needle, filtrated through nylon meshes and centrifugated for $5 \mathrm{~min}$ at 1,200 $\mathrm{rpm}$. Isolated $\mathrm{BM}$ cells were then incubated in $\mathrm{RBC}$ lysis solution $\left(0.15 \mathrm{M} \mathrm{NH}_{4} \mathrm{Cl}, 10 \mathrm{mM}\right.$ $\mathrm{NaHCO}_{3}, 10 \mathrm{mM}$ EDTA) and washed twice with phosphatebuffered saline (PBS). The cells were then plated at $1 \times 10^{7}$ cells/ 100 culture dishes in Iscove's Modified Dulbecco's Medium (IMDM; Sigma, St. Louis, MO) with 10\% heat-inactivated 
fetal bovine serum (FBS). After $48 \mathrm{~h}$, non-adherent cells were removed via aspiration, and the Mesencult basal medium with $10 \%$ mesenchymal stem cell stimulatory supplement (Stem Cell Technologies, Vancouver, Canada; cat. nos. 05501 and 05502) was replenished. Three weeks from the BM isolation, all MSCs used in this study were at 4 passages.

Adipogenic differentiation. MSCs cultured for 3 weeks following primary culture were plated on Mesencult basal medium containing $10 \%$ adipogenic stimulatory supplement (Stem Celfigl Technologies; cat. no. 05401). The medium was replenished once every 3 days for 2 weeks.

Liver cell isolation. Six-week-old mice were injected once a day with $10 \% \mathrm{CCl}_{4}(10 \mu 1$ per gram) in mineral oil via the intraperitoneal route for two consecutive days. After 1 week, the mice were sacrificed and their liver tissues were collected aseptically followed by $30 \mathrm{~min}$ of collagenase treatment $(0.5 \mathrm{mg} / \mathrm{ml}$ in RPMI-1640 with $10 \% \mathrm{FBS}$; Roche, Indianapolis, IN) at $37^{\circ} \mathrm{C}$. Liver cells were isolated from the collagenasetreated liver tissue following filtration through nylon meshes. For the co-culturing of liver cells and MSCs, the MSCs $\left(5 \times 10^{5}\right.$ cells/well) were plated onto the lower chambers of transwell culture plates (Falcon, Bedford, MA), while the liver cells $\left(3 \times 10^{5} /\right.$ well $)$ were introduced into the inserted upperchamber ( $3 \mu \mathrm{m}$ in pore size) with Dulbecco's modified Eagle's medium (Fig. 1H). After $48 \mathrm{~h}$ of co-culture, the inserted upper chambers were removed, and the MSCs in the lower chamber were collected for RNA isolation.

Flow cytometry. MSCs were analyzed for cell surface marker expression after culturing for 3 weeks. The cells were washed with PBS and stained at $4^{\circ} \mathrm{C}$ for 30 min with a combination of the following antibodies: FITC-anti-mouse CD106, FITC-anti-mouse CD34, FITC-anti-mouse CD31, PE-anti-mouse CD73, PE-anti-mouse CD105, PE-anti-mouse CD45. All antibodies were purchased from BD Pharmingen with the exception of PE-anti-mouse CD105, which was obtained from R\&D Systems (Minneapolis, MN). Flow cytometric analysis was performed using FACSCalibur and CellQuest software (BD).

Tissue preparation. The liver was perfused via the heart with $4 \%$ paraformaldehyde to flush out blood cells, then incubated with $4 \%$ paraformaldehyde overnight at room temperature for fixation. After washing twice with water, fixed livers were stored in $70 \%$ ethanol at $4^{\circ} \mathrm{C}$ and embedded in paraffin. Sections were stained with hematoxylin and eosin.

\section{Cell staining}

Albumin staining. MSCs co-cultured with liver cells or not, as described above, were fixed for $10 \mathrm{~min}$ in ice-cold methanol. The cells were treated with $1 \%$ BSA in PBST (0.05\% Tween-20 in PBS) for 30 min to block the unspecific binding of antibodies, then incubated overnight in primary antibody against albumin (Abcam; cat. no. ab19196) at $4^{\circ} \mathrm{C}$ (1:800). The next day, the secondary antibody, biotinylated anti-rabbit IgG (DakoCytomation, Denmark) was applied for $30 \mathrm{~min}$. Albumin expression was detected via the streptovidinHRP/DAB substrate (DakoCytomation) reaction.

Giemsa staining. After 14 days of MSC culture, colony forming units of fibroblasts (CFU-F) were assessed after Giemsa staining. Cells fixed in methanol for $5 \mathrm{~min}$ were completely dried, and Giemsa stain solution was added.
Adipocyte staining. Adipocytes differentiated from MSCs were stained with Oil Red O solution. First, the cells were fixed for $5 \mathrm{~min}$ in $10 \%$ formalin and incubated for $1 \mathrm{~h}$ in newly changed $10 \%$ formalin. After washing with $60 \%$ isopropanol, the cells were dried completely and treated for $1 \mathrm{~h}$ with Oil Red O solution.

Isolation of total RNA. Total RNA was extracted from adherent MSCs co-cultured with normal liver tissue or liver tissue from $\mathrm{CCl}_{4}$-injected mice, as well as from MSCs without co-culture, using TRIzol solution (Invitrogen, Carlsbad, CA) in accordance with the manufacturer's instructions. RNA samples were stored at $-70^{\circ} \mathrm{C}$ until future use.

Microarray analysis. Two-color microarray-based Agilent chips containing 44,000 mouse genes (Digital Genomics, Seoul, Korea) were utilized. Total RNA $(50 \mu \mathrm{g})$ was prepared from 3 groups of MSCs: the MSC group (untreated MSCs), the liver group (from MSCs co-cultured with normal liver cells) and the $\mathrm{CCl}_{4}$ liver group (from MSCs co-cultured with the livers of $\mathrm{CCl}_{4}$-injected mice). Samples from the MSC group were labeled with Cy5 while samples from the liver group were labeled with $\mathrm{Cy} 3$, and RNA samples from the MSC group were utilized as RNA references for the comparison of gene expression profiles. These microarray experiments were repeated three times for the different RNA batches. Genes were selected on the basis of differential $\mathrm{Cy} 3 / \mathrm{Cy} 5$ expression ratios $\geq 2$ in response. Gene clustering was generated from the gene expression data using Cluster and TreeView software (Eisen Lab; http://rana.lbl.gov/EisenSoftware.htm). Additional filtering was applied with a 2-fold change minimum to analyze the genes expressed in mouse liver tissue using Ingenuity Pathway Analysis (IPA) software (Ingenuity Systems, Redwood City, CA; http://www.ingenuity.com/).

\section{Results}

Before the liver cells from $\mathrm{CCl}_{4}$-injected mice were isolated, liver tissue from the normal (control) and $\mathrm{CCl}_{4}$-injected mice was fixed and stained with $\mathrm{H} \& \mathrm{E}$ (Fig. 1) so that differences between the two groups might be examined. Fig. 1A and B show characteristic features of the mouse liver, such as anisocytosis (an uneven size of liver cells) and anisokaryosis (an uneven size of liver cell nuclei). In Fig. 1B, a liver section from a $\mathrm{CCl}_{4}$-injected mouse exhibits vacuolization of the liver cells, indicating that the cells were damaged by the $\mathrm{CCl}_{4}$ injection.

In order to characterize the MSCs from the liver group, a CFU-F assay was performed (Fig. 2B) and the isolated MSCs were induced to differentiate into adipocytes (Fig. 2C) to confirm their multipotential differentiation. Additionally, CD73, CD105 and CD106 expression, as well as the negative expression of CD31, CD34 and CD45, was observed (Fig. 2D).

To assess the gene expression profiles of the MSCs from the liver and $\mathrm{CCl}_{4}$ liver groups, we co-cultured liver cells in the upper chamber and MSCs in the lower chamber of transwell plates (Fig. 3). After $48 \mathrm{~h}$ of transwell culture, the upper chamber was removed and the cells from the lower chamber, containing MSCs stimulated by liver cells or injured liver cells, were collected. Next, RNAs from the cells of the lower chamber were isolated. Co-culture and RNA isolation was 

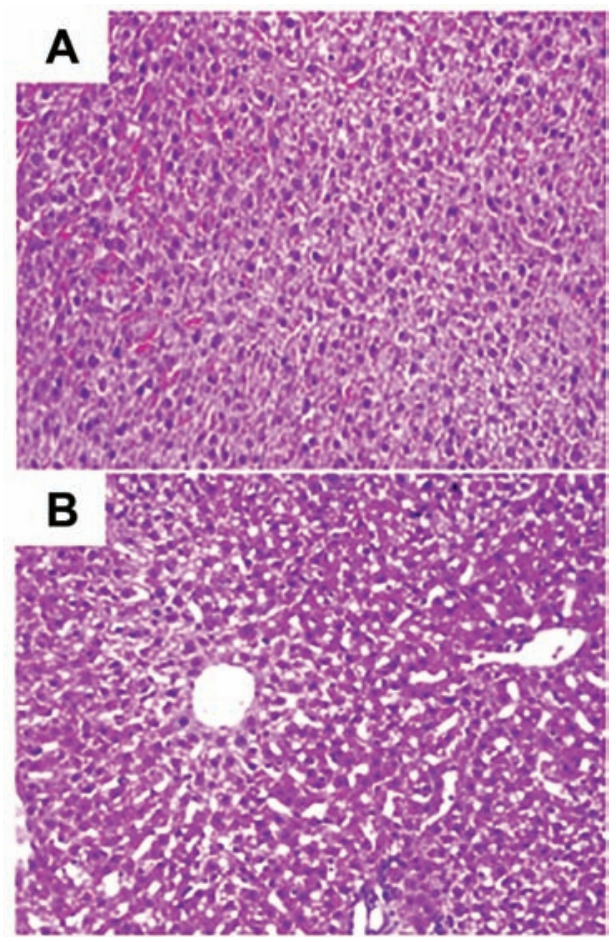

Figure 1. Representative sections of liver tissue stained with hematoxylin and eosin. (A) Normal liver. (B) Injured liver from a mouse 8 days after 2 consecutive injections of $\mathrm{CCl}_{4}$. White punctuated space among the liver cells from $\mathrm{CCl}_{4}$-injected mice can be seen. (Original magnification, $\mathrm{x} 200$ ).

repeated three times for each of the experimental groups, and the expression profile was presented as the mean values from these three microchip assay analyses.

Genes upregulated in the liver group as compared with the untreated MSC group are listed in Table I. Genes upregulated in the $\mathrm{CCl}_{4}$ liver group as compared with the MSC group are shown in Table II. In addition, Tables III and IV show IPA-filtered genes that evidenced a $>2$-fold change in the gene expression of MSCs from the liver and $\mathrm{CCl}_{4}$ liver groups, respectively. The results of microarray analysis of MSCs from the liver group demonstrate that inhibitor of DNA binding 1, Forkhead box G1, Wnt2, CD34, CXCR6 (receptor for CXCL16), tissue inhibitor of metalloproteinase 3, periostin, procollagen type 1 , IL-2, neuregulin 4, CCR3 (receptor for RANTES, MCP-2, -3, -4), IL-11, CD74 (Ii chain of class II MHC molecules) and catenins were upregulated. When co-cultured with the $\mathrm{CCl}_{4}$-injured liver cells, the expression of RAR-related orphan receptor $\beta$, retinoic acid receptor $\beta$, forkhead box $G 1$, neural cell adhesion molecule 1, matrix metallopeptidase 12, insulin-like growth factor binding protein 6 , nerve growth factor receptor, chordin, CXCL2, cytoglobin, erythropoietin, v-Erb, hypoxia inducible factor 3 ( $\alpha$ subunit), Vav2 and hepatic nuclear factor $4 \alpha$ was upregulated.

Immunostaining analysis of albumin was conducted on MSCs co-cultured with liver cells from mice treated or untreated with $\mathrm{CCl}_{4}$. After $48 \mathrm{~h}$ of co-culture with $\mathrm{CCl}_{4}$-treated liver cells, the MSCs expressed albumin (Fig. 4). Therefore, MSCs derived from BM cells seemed to differentiate into functional hepatocytes under the specific conditions provided by a damaged liver.
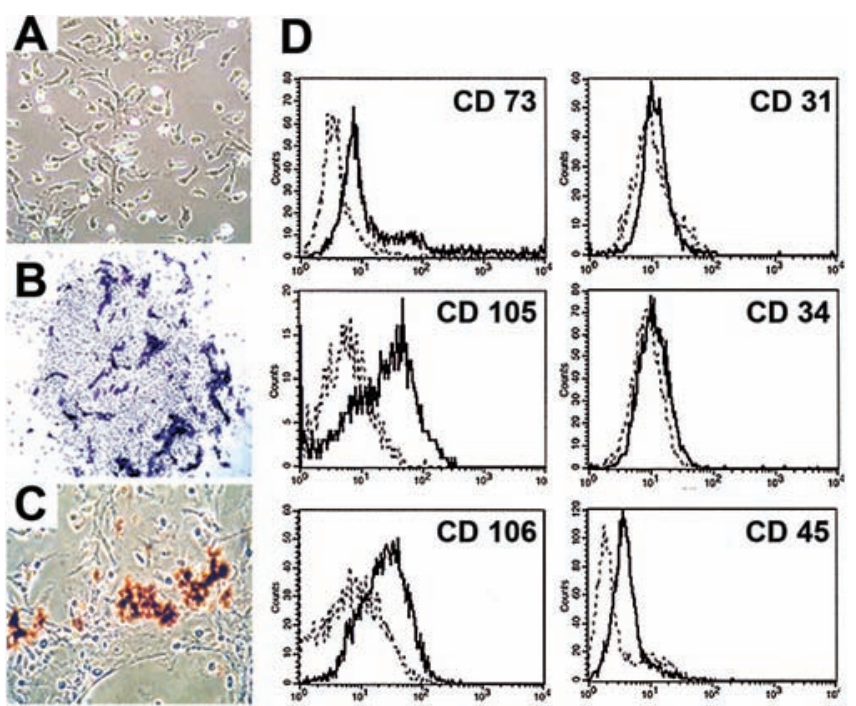

Figure 2. Preparation and characterization of isolated MSCs. The newly planted bone marrow cells were divided into adherent and non-adherent cells after $48 \mathrm{~h}$ of culture. (A) Adherent cells, or MSCs, proliferated and formed CFU-F. (B) This was confirmed by Giemsa staining on day 14. (C) MSCs cultured for 3 weeks were differentiated into adipocytes and stained red with Oil Red O (original magnification A-C, x100). (D) For the phenotypic markers, the MSC markers (CD73, CD105 and CD106) were positive. In contrast, the hematopoietic (CD34 and CD45) and endothelial (CD31) cell markers were not detectable in the cells after 21 days of culture.
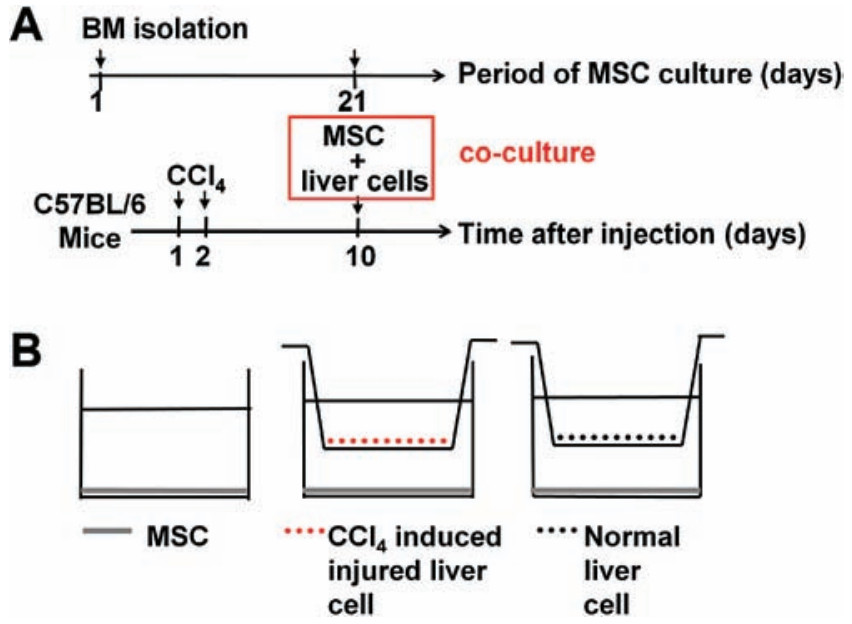

Figure 3. Co-culturing of MSCs and liver cells in transwell plates. (A) MSCs and injured livers were established according to the indicated co-culture schedule. (B) MSCs co-cultured with normal or $\mathrm{CCl}_{4}$-injured liver cells on the transwell insert co-culture system.

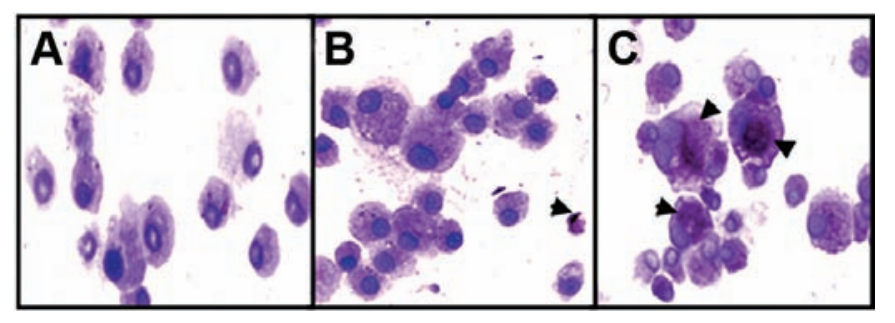

Figure 4. Albumin detection in co-cultured MSCs by immunocytochemistry. MSCs cultured alone (A) or co-cultured with normal liver cells (B) did not express albumin. However, MSCs co-cultured with $\mathrm{CCl}_{4}$-injected liver cells (C) evidenced albumin positivity. 
Table I. Genes upregulated in MSCs co-cultured with normal liver cells.

\begin{tabular}{|c|c|c|c|}
\hline Accession & Symbol & Name & Mean \\
\hline NM_207624 & $A C E$ & $\begin{array}{l}\text { Mus musculus angiotensin converting enzyme } \\
\text { (Ace), transcript variant 1, mRNA [NM_207624] }\end{array}$ & 2.21 \\
\hline NM_007392 & ACTA2 & Actin, $\alpha 2$, smooth muscle, aorta & 2.64 \\
\hline NM_007395 & $A C V R I B$ & Activin A receptor, type 1B & 2.09 \\
\hline NM_009633 & $A D R A 2 B$ & Adrenergic receptor, $\alpha 2 b$ & 2.78 \\
\hline NM_011784 & AGTRL1 & Angiotensin receptor-like 1 & 2.59 \\
\hline NM_011784 & AGTRL1 & Angiotensin receptor-like 1 & 2.34 \\
\hline NM_009914 & $C C R 3$ & Chemokine (C-C motif) receptor 3 & 2.08 \\
\hline NM_133654 & $C D 34$ & CD34 antigen & 4.22 \\
\hline NM_010545 & $C D 74$ & $\begin{array}{l}\text { CD74 antigen (invariant polypeptide of major } \\
\text { histocompatibility complex, class II antigen-associated) }\end{array}$ & 2.19 \\
\hline NM_007664 & $\mathrm{CDH} 2$ & Cadherin 2 & 2.01 \\
\hline NM_007664 & $\mathrm{CDH} 2$ & Cadherin 2 & 2.01 \\
\hline NM_007693 & $C H G A$ & Chromogranin A & 2.31 \\
\hline NM_016673 & CNTFR & Ciliary neurotrophic factor receptor & 2.84 \\
\hline NM_007742 & COLIAl & Procollagen, type I, $\alpha 1$ & 3.22 \\
\hline NM_007743 & COLIA2 & Procollagen, type I, $\alpha 2$ & 2.14 \\
\hline AK076297 & COL27Al & Procollagen, type XXVII, $\alpha 1$ & 2.27 \\
\hline AK008121 & CTNNA1 & Catenin (cadherin associated protein), $\alpha 1$ & 2.41 \\
\hline AK077879 & CTNNAL1 & Catenin (cadherin associated protein), $\beta 1$ & 2.92 \\
\hline NM_030712 & CXCR6 & Chemokine (C-X-C motif) receptor 6 & 3.40 \\
\hline NM_030206 & $C Y G B$ & Mus musculus cytoglobin & 2.49 \\
\hline NM_009998 & $C Y P 2 B 10$ & Cytochrome $\mathrm{P} 450$, family 2 , subfamily b, polypeptide 10 & 2.03 \\
\hline NM_007822 & CYP4A14 & Cytochrome P450, family 4, subfamily a, polypeptide 14 & 2.32 \\
\hline NM_007824 & CYP7A1 & Cytochrome P450, family 7, subfamily a, polypeptide 1 & 2.04 \\
\hline NM_010045 & $D A R C$ & Duffy blood group, chemokine receptor & 2.52 \\
\hline NM_010106 & EEF1A1 & Eukaryotic translation elongation factor $1 \alpha 1$ & 2.12 \\
\hline NM_010109 & EFNA5 & Ephrin A5 & 2.13 \\
\hline NM_207667 & $F G F 14$ & Fibroblast growth factor 15 & 2.04 \\
\hline NM_008005 & $F G F 18$ & Fibroblast growth factor 2 & 2.44 \\
\hline AK005502 & FLT1 & FMS-like tyrosine kinase 1 & 2.06 \\
\hline AK034946 & FMO1 & Flavin containing monooxygenase 1 & 2.13 \\
\hline NM_008241 & FOXG1 & Forkhead box G1 & 4.55 \\
\hline NM_008126 & GJB3 & Gap junction membrane channel protein $B 3$ & 2.80 \\
\hline NM_008127 & GJB4 & Gap junction membrane channel protein $\beta 4$ & 2.44 \\
\hline NM_013920 & $H N F 4 G$ & Hepatocyte nuclear factor $4, \gamma$ & 2.55 \\
\hline NM_008296 & $H S F 1$ & Heat shock factor 1 & 2.53 \\
\hline NM_010495 & IDI & Inhibitor of DNA binding 1 & 4.69 \\
\hline NM_008344 & IGFBP6 & Insulin-like growth factor binding protein 6 & 3.19 \\
\hline NM_008350 & $I L-11$ & Interleukin 11 & 3.13 \\
\hline NM_019451 & $I L-1 F 5$ & Interleukin 1 family, member $5(\delta)$ & 2.45 \\
\hline NM_008366 & $I L-2$ & Interleukin 2 & 3.20 \\
\hline NM_008368 & $I L-2 R B$ & Interleukin 2 receptor, $\beta$ chain & 3.03 \\
\hline NM_013565 & ITGA3 & Integrin $\alpha 3$ & 2.00 \\
\hline NM_008501 & $L I F$ & Leukemia inhibitory factor & 2.32 \\
\hline NM_009158 & МАРК 10 & Mitogen activated protein kinase 10 & 2.08 \\
\hline NM_008598 & $M G M T$ & O-6-methylguanine-DNA methyltransferase & 2.03 \\
\hline NM_011846 & MMP17 & Matrix metallopeptidase 17 & 2.14 \\
\hline NM_010808 & $M M P 24$ & Matrix metallopeptidase 24 & 2.01 \\
\hline AK038264 & MSH3 & MutS homolog 3 (E. coli) & 2.36 \\
\hline NM_008634 & MTAPIB & Microtubule-associated protein $1 \mathrm{~B}$ & 2.37 \\
\hline NM_013607 & MYH11 & Myosin, heavy polypeptide 11 , smooth muscle & 2.21 \\
\hline NM_013607 & MYH11 & Myosin, heavy polypeptide 11 , smooth muscle & 2.21 \\
\hline
\end{tabular}


Table I. Continued.

\begin{tabular}{|c|c|c|c|}
\hline Accession & Symbol & Name & Mean \\
\hline $\mathrm{X} 15052$ & NCAM1 & Neural cell adhesion molecule 1 & 2.25 \\
\hline NM_010875 & NCAM1 & Neural cell adhesion molecule 1 & 2.14 \\
\hline NM_010900 & NFATC2IP & $\begin{array}{l}\text { Nuclear factor of activated T-cells, cytoplasmic, } \\
\text { calcineurin-dependent } 2 \text { interacting protein }\end{array}$ & 2.10 \\
\hline NM_008700 & $N K X 2-5$ & NK2 transcription factor related, locus 5 (Drosophila) & 2.60 \\
\hline AK012322 & $N R 2 F 2$ & Nuclear receptor subfamily 2 , group F, member 2 & 2.91 \\
\hline X76653 & $N R 2 F 2$ & Nuclear receptor subfamily 2 , group F, member 2 & 2.40 \\
\hline NM_032002 & $N R G 4$ & Neuregulin 4 & 3.19 \\
\hline NM_008814 & $P D X 1$ & Pancreatic and duodenal homeobox 1 & 2.30 \\
\hline NM_015784 & POSTN & Periostin, osteoblast specific factor & 3.28 \\
\hline NM_008969 & PTGS1 & Prostaglandin-endoperoxide synthase 1 & 2.31 \\
\hline NM_008973 & $P T N$ & Pleiotrophin & 2.76 \\
\hline NM_011243 & $R A R B$ & Retinoic acid receptor, $ß$ & 3.06 \\
\hline NM_009084 & RPL37A & Ribosomal protein L37a & 2.10 \\
\hline NM_009115 & $S 100 B$ & S100 protein, $ß$ polypeptide, neural & 2.55 \\
\hline NM_011347 & SELP & Selectin, platelet & 2.07 \\
\hline AK038807 & SLIT2 & slit homolog 2 (Drosophila) & 2.20 \\
\hline AK020817 & SMUG1 & Single-strand selective monofunctional uracil DNA glycosylase & 2.14 \\
\hline NM_009235 & SOX15 & SRY-box containing gene 15 & 2.00 \\
\hline NM_011443 & SOX2 & SRY-box containing gene 2 & 2.56 \\
\hline NM_025285 & STMN2 & Stathmin-like 2 & 3.03 \\
\hline NM_019507 & $T B X 21$ & T-box 21 & 2.20 \\
\hline NM_011595 & TIMP3 & Tissue inhibitor of metalloproteinase 3 & 3.31 \\
\hline NM_021406 & TREM1 & Triggering receptor expressed on myeloid cells 1 & 2.22 \\
\hline AK048623 & TRP63 & Transformation related protein 63 & 2.19 \\
\hline AK006986 & $T Y K 2$ & Tyrosine kinase 2 & 2.70 \\
\hline NM_011707 & $V T N$ & Vitronectin & 2.07 \\
\hline NM_023653 & WNT2 & Wingless-related MMTV integration site 2 & 4.22 \\
\hline NM_009523 & WNT4 & Wingless-related MMTV integration site 4 & 2.56 \\
\hline
\end{tabular}

Table II. Genes upregulated in MSCs co-cultured with $\mathrm{CCl}_{4}$-injured liver cells.

\begin{tabular}{|c|c|c|c|}
\hline Accession & Symbol & Name & Mean \\
\hline AK051467 & $A G T P B P 1$ & ATP/GTP binding protein 1 & 2.07 \\
\hline NM_011784 & AGTRL1 & Angiotensin receptor-like 1 & 2.33 \\
\hline NM_019577 & CCL24 & Chemokine (C-C motif) ligand 24 & 2.37 \\
\hline NM_009139 & CCL6 & Chemokine (C-C motif) ligand 6 & 2.26 \\
\hline NM_133654 & $C D 34$ & CD34 antigen & 2.56 \\
\hline NM_007650 & CD5 & CD5 antigen & 2.33 \\
\hline NM_009873 & $C D K 6$ & Cyclin-dependent kinase 6 & 2.80 \\
\hline NM_009893 & CHRD & Chordin & 3.08 \\
\hline AK003879 & COL27Al & Procollagen, type XXVII, $\alpha 1$ & 2.57 \\
\hline NM_007758 & $C R 2$ & Complement receptor 2 & 2.39 \\
\hline NM_008176 & CXCL2 & Chemokine (C-X-C motif) ligand 2 & 2.60 \\
\hline NM_030206 & $C Y G B$ & Cytoglobin & 4.90 \\
\hline NM_201640 & CYP4A10 & Cytochrome P450, family 4 , subfamily a, polypeptide 10 & 2.09 \\
\hline NM_007824 & CYP7A1 & Cytochrome P450, family 7, subfamily a, polypeptide 1 & 2.29 \\
\hline AK009701 & $D A P K 1$ & Death associated protein kinase 1 & 2.08 \\
\hline NM_010109 & EFNA5 & Ephrin A5 & 2.04 \\
\hline NM_207655 & $E G F R$ & Epidermal growth factor receptor & 2.23 \\
\hline
\end{tabular}


Table II. Continued.

\begin{tabular}{|c|c|c|c|}
\hline Accession & Symbol & Name & Mean \\
\hline NM_007942 & $E P O$ & Erythropoietin & 2.73 \\
\hline XM_136682 & ERBB4 & v-Erb-a erythroblastic leukemia viral oncogene homolog 4 (avian) & 2.46 \\
\hline NM_021272 & $F A B P 7$ & Fibroblast growth factor 1 & 2.22 \\
\hline NM_207667 & FGF14 & Fibroblast growth factor 15 & 2.42 \\
\hline NM_008005 & FGF18 & Fibroblast growth factor 2 & 2.90 \\
\hline AK008922 & $F G F 22$ & Fibroblast growth factor 23 & 2.32 \\
\hline NM_008011 & FGFR4 & Fibroblast growth factor receptor 4 & 2.17 \\
\hline NM_008241 & FOXG1 & Forkhead box G1 & 3.46 \\
\hline NM_008055 & FZD4 & Frizzled homolog 4 (Drosophila) & 2.52 \\
\hline NM_008107 & GDF1 & Growth differentiation factor 1 & 2.06 \\
\hline NM_008160 & GPXI & Glutathione peroxidase & 2.06 \\
\hline AK002213 & GSTM1 & Glutathione S-transferase, mu 1 & 2.63 \\
\hline NM_016868 & HIF3A & Hypoxia inducible factor $3, \alpha$ subunit & 2.22 \\
\hline NM_008261 & $H N F 4 A$ & Hepatic nuclear factor $4, \alpha$ & 3.03 \\
\hline NM_010495 & IDI & Inhibitor of DNA binding 1, dominant negative helix-loop-helix protein & 2.11 \\
\hline NM_010496 & ID2 & Inhibitor of DNA binding 2, dominant negative helix-loop-helix protein & 2.10 \\
\hline NM_008344 & IGFBP6 & Insulin-like growth factor binding protein 6 & 3.18 \\
\hline NM_019451 & IL1F5 & Interleukin 1 family, member $5(\delta)$ & 2.37 \\
\hline NM_001005608 & ITGB4 & Integrin $\beta 4$ & 2.00 \\
\hline XM_140451 & $L A M A 3$ & Laminin, $\alpha 3$ & 2.27 \\
\hline ВС070467 & $M A P 2 K 7$ & Mitogen activated protein kinase kinase 7 & 2.02 \\
\hline AK053819 & MAPK8IP1 & Mitogen activated protein kinase 8 interacting protein 1 & 2.30 \\
\hline NM_008605 & MMP12 & Matrix metallopeptidase 12 & 3.34 \\
\hline NM_008611 & MMP8 & Matrix metallopeptidase 8 & 2.22 \\
\hline NM_133250 & MUTYH & MutY homolog (E.coli) & 2.06 \\
\hline AK048336 & MYST4 & MYST histone acetyltransferase monocytic leukemia 4 & 2.08 \\
\hline X15052 & NCAMI & Neural cell adhesion molecule 1 & 3.36 \\
\hline NM_010896 & NEUROG1 & Neurogenin 1 & 2.14 \\
\hline NM_033217 & $N G F R$ & Nerve growth factor receptor (TNFR superfamily, member 16) & 3.11 \\
\hline NM_008725 & $N P P A$ & Natriuretic peptide precursor type A & 2.49 \\
\hline AK041047 & NRIDI & Nuclear receptor subfamily 1 , group $\mathrm{D}$, member 1 & 2.22 \\
\hline X76653 & $N R 2 F 2$ & Nuclear receptor subfamily 2 , group F, member 2 & 2.61 \\
\hline AK012322 & $N R 2 F 2$ & Nuclear receptor subfamily 2 , group F, member 2 & 2.58 \\
\hline NM_013630 & $P K D 1$ & Polycystic kidney disease 1 homolog & 2.09 \\
\hline AK040305 & POLI & Polymerase (DNA directed),, & 2.00 \\
\hline ВC042707 & PROK1 & Prokineticin 1 & 2.37 \\
\hline NM_008973 & $P T N$ & Pleiotrophin & 2.54 \\
\hline NM_011243 & $R A R B$ & Retinoic acid receptor, $B$ & 4.88 \\
\hline NM_011244 & $R A R G$ & Retinoic acid receptor, $\gamma$ & 2.05 \\
\hline NM_134257 & $R G S 3$ & Regulator of G-protein signaling 3 & 2.09 \\
\hline ВС058269 & $R O R B$ & RAR-related orphan receptor $\beta$ & 5.32 \\
\hline NM_009115 & $S 100 B$ & S100 protein, $ß$ polypeptide, neural & 2.31 \\
\hline AK038807 & SLIT2 & slit homolog 2 (Drosophila) & 3.00 \\
\hline AK090367 & SMUG1 & Single-strand selective monofunctional uracil DNA glycosylase & 2.56 \\
\hline NM_011445 & SOX6 & SRY-box containing gene 6 & 2.93 \\
\hline NM_011448 & SOX9 & SRY-box containing gene 9 & 2.73 \\
\hline NM_009291 & STRA6 & Stimulated by retinoic acid gene 6 & 2.21 \\
\hline NM_011578 & $T G F B R 3$ & Transforming growth factor, $B$ receptor III & 2.00 \\
\hline NM_009380 & $T H R B$ & Thyroid hormone receptor $\beta$ & 2.95 \\
\hline AK006986 & TYK2 & Tyrosine kinase 2 & 2.46 \\
\hline NM_009500 & $V A V 2$ & Vav 2 oncogene & 2.14 \\
\hline M89800 & WNT6 & Wingless-related MMTV integration site 6 & 2.34 \\
\hline
\end{tabular}


Table III. Gene expression profiles in MSCs co-cultured with normal liver cells.

\begin{tabular}{|c|c|c|c|c|}
\hline Symbol & Name & Location & Accession & Fold change \\
\hline$A C E$ & $\begin{array}{l}\text { Angiotensin I converting enzyme } \\
\text { (peptidyl-dipeptidase A) } 1\end{array}$ & Plasma membrane & NM_207624 & 2.206 \\
\hline $\begin{array}{l}\text { АCTA2 } \\
\text { (includes EG:59) }\end{array}$ & Actin, $\alpha 2$, smooth muscle, aorta & Cytoplasm & NM_007392 & 2.642 \\
\hline$A C V R 1 B$ & Activin A receptor, type IB & Plasma membrane & NM_007395 & 2.092 \\
\hline ADAMTS1 & $\begin{array}{l}\text { ADAM metallopeptidase with } \\
\text { thrombospondin type } 1 \text { motif, } 1\end{array}$ & Extracellular space & NM_009621 & -3.448 \\
\hline ADAMTS5 & $\begin{array}{l}\text { ADAM metallopeptidase with thrombospondin } \\
\text { type } 1 \text { motif, } 5 \text { (aggrecanase-2) }\end{array}$ & Extracellular space & AK046558 & -3.19 \\
\hline$A P A F 1$ & Apoptotic peptidase activating factor 1 & Cytoplasm & NM_009684 & -2.001 \\
\hline BCL10 & B-cell CLL/lymphoma 10 & Cytoplasm & AK080820 & -2.157 \\
\hline$B C L 2 L 11$ & BCL2-like 11 (apoptosis facilitator) & Cytoplasm & NM_207680 & -2.062 \\
\hline C3 & Complement component 3 & Extracellular space & NM_009778 & -6.849 \\
\hline CASP3 & Caspase 3 , apoptosis-related cysteine peptidase & Cytoplasm & NM_009810 & -2.423 \\
\hline$C A V 1$ & Caveolin 1 , caveolae protein, $22 \mathrm{kDa}$ & Plasma membrane & NM_007616 & -2.479 \\
\hline CD34 & CD34 molecule & Plasma membrane & NM_133654 & 4.217 \\
\hline$C D 36$ & CD36 molecule (thrombospondin receptor) & Plasma membrane & NM_007643 & -2.174 \\
\hline$C D 74$ & $\begin{array}{l}\text { CD74 molecule, major histocompatibility } \\
\text { complex, class II invariant chain }\end{array}$ & Plasma membrane & NM_010545 & 2.192 \\
\hline$C D 86$ & CD86 molecule & Plasma membrane & NM_019388 & -2.618 \\
\hline$C D C 42$ & $\begin{array}{l}\text { Cell division cycle } 42 \\
\text { (GTP binding protein, } 25 \mathrm{kDa} \text { ) }\end{array}$ & Cytoplasm & NM_009861 & -2.217 \\
\hline $\mathrm{CDH} 2$ & Cadherin 2, type 1, N-cadherin (neuronal) & Plasma membrane & NM_007664 & 2.012 \\
\hline CEACAMI & $\begin{array}{l}\text { Carcinoembryonic antigen-related cell } \\
\text { adhesion molecule } 1 \text { (biliary glycoprotein) }\end{array}$ & Plasma membrane & NM_011926 & -2.506 \\
\hline CFLAR & CASP8 and FADD-like apoptosis regulator & Cytoplasm & NM_009805 & -2.389 \\
\hline CNTFR & Ciliary neurotrophic factor receptor & Plasma membrane & NM_016673 & 2.836 \\
\hline COLI8A1 & Collagen, type XVIII, $\alpha 1$ & Extracellular space & NM_009929 & -2.326 \\
\hline COL1A1 & Collagen, type I, $\alpha 1$ & Extracellular space & NM_007742 & 3.218 \\
\hline COL1A2 & Collagen, type I, $\alpha 2$ & Extracellular space & NM_007743 & 2.143 \\
\hline COL27A1 & Collagen, type XXVII, $\alpha 1$ & Extracellular space & AK076297 & 2.270 \\
\hline CUL1 & Cullin 1 & Nucleus & NM_012042 & -3.055 \\
\hline CXCL3 & Chemokine (C-X-C motif) ligand 3 & Extracellular space & NM_009140 & -2.439 \\
\hline CXCL12 & $\begin{array}{l}\text { Chemokine (C-X-C motif) ligand } 12 \\
\text { (stromal cell-derived factor } 1 \text { ) }\end{array}$ & Extracellular space & NM_013655 & -2.329 \\
\hline $\begin{array}{l}\text { CXCR6 } \\
\text { (includes EG:10663) }\end{array}$ & Chemokine (C-X-C motif) receptor 6 & Plasma membrane & NM_030712 & 3.400 \\
\hline $\begin{array}{l}\text { CYP2B6 } \\
\text { (includes EG:1555) }\end{array}$ & $\begin{array}{l}\text { Cytochrome P450, family } 2 \text {, subfamily B, } \\
\text { polypeptide } 6\end{array}$ & Cytoplasm & NM_009998 & 2.027 \\
\hline EEFIAI & Eukaryotic translation elongation factor $1 \alpha 1$ & Cytoplasm & NM_010106 & 2.120 \\
\hline EFNA5 & Ephrin-A5 & Plasma membrane & NM_010109 & 2.130 \\
\hline$E R B B 2 I P$ & erbb2 interacting protein & Extracellular space & NM_001005868 & -2.107 \\
\hline FN1 & Fibronectin 1 & Plasma membrane & NM_010233 & -2.439 \\
\hline GNA13 & $\begin{array}{l}\text { Guanine nucleotide binding protein } \\
\text { (G protein), } \alpha 13\end{array}$ & Plasma membrane & NM_010303 & -2.128 \\
\hline$H G F$ & $\begin{array}{l}\text { Hepatocyte growth factor } \\
\text { (hepapoietin A; scatter factor) }\end{array}$ & Extracellular space & NM_010427 & -2.439 \\
\hline IDI & $\begin{array}{l}\text { Inhibitor of DNA binding 1, dominant } \\
\text { negative helix-loop-helix protein }\end{array}$ & Nucleus & NM_010495 & 4.690 \\
\hline$I G F 2$ & Insulin-like growth factor 2 (somatomedin A) & Extracellular space & NM_010514 & -2.528 \\
\hline IGFBPG & Insulin-like growth factor binding protein 6 & Extracellular space & NM_008344 & 3.192 \\
\hline$I L 1 B$ & Interleukin $1, \beta$ & Extracellular space & NM_008361 & -3.906 \\
\hline ILIRN & Interleukin 1 receptor antagonist & Extracellular space & NM_031167 & -2.924 \\
\hline
\end{tabular}


Table III. Continued.

\begin{tabular}{|c|c|c|c|c|}
\hline Symbol & Name & Location & Accession & Fold change \\
\hline ITGA3 & $\begin{array}{l}\text { Integrin, } \alpha 3 \text { (antigen CD } 49 C \text {, } \\
\alpha 3 \text { subunit of VLA-3 receptor) }\end{array}$ & Plasma membrane & NM_013565 & 2.002 \\
\hline ITGA6 & Integrin, $\alpha 6$ & Plasma membrane & AK045391 & -2.551 \\
\hline $\begin{array}{l}\text { KITLG } \\
\text { (includes EG:4254) }\end{array}$ & KIT ligand & Extracellular space & NM_013598 & -2.171 \\
\hline MAF & $\begin{array}{l}\text { v-maf musculoaponeurotic fibrosarcoma } \\
\text { oncogene homolog (avian) }\end{array}$ & Nucleus & NM_001025577 & -2.309 \\
\hline МАРК & Mitogen-activated protein kinase 9 & Cytoplasm & NM_207692 & -2.003 \\
\hline MCL1 & Myeloid cell leukemia sequence 1 (BCL2-related) & Cytoplasm & NM_008562 & -2.547 \\
\hline MMP17 & Matrix metallopeptidase 17 (membrane-inserted) & Extracellular space & NM_011846 & 2.142 \\
\hline$M M P 24$ & Matrix metallopeptidase 24 (membrane-inserted) & Extracellular space & NM_010808 & 2.012 \\
\hline NFATC2 & $\begin{array}{l}\text { Nuclear factor of activated T-cells, } \\
\text { cytoplasmic, calcineurin-dependent } 2\end{array}$ & Nucleus & AK081853 & -2.237 \\
\hline$N R 3 C 1$ & $\begin{array}{l}\text { Nuclear receptor subfamily } 3 \text {, group C, } \\
\text { member } 1 \text { (glucocorticoid receptor) }\end{array}$ & Nucleus & NM_008173 & -2.134 \\
\hline PTGS1 & $\begin{array}{l}\text { Prostaglandin-endoperoxide synthase } 1 \\
\text { (prostaglandin G/H synthase and cyclooxygenase) }\end{array}$ & Cytoplasm & NM_008969 & 2.310 \\
\hline PTPRC & Protein tyrosine phosphatase, receptor type, C & Plasma membrane & NM_011210 & -3.067 \\
\hline$P T X 3$ & Pentraxin-related gene, rapidly induced by IL-1 $ß$ & Extracellular space & NM_008987 & -2.182 \\
\hline$R A R B$ & Retinoic acid receptor, $\beta$ & Nucleus & NM_011243 & 3.058 \\
\hline SELP & $\begin{array}{l}\text { Selectin P (granule membrane protein } \\
140 \mathrm{kDa} \text {, antigen CD62) }\end{array}$ & Plasma membrane & NM_011347 & 2.070 \\
\hline SLIT2 & Slit homolog 2 (Drosophila) & Extracellular space & AK038807 & 2.200 \\
\hline SOCS3 & Suppressor of cytokine signaling 3 & Cytoplasm & NM_007707 & -2.268 \\
\hline$S P P 1$ & $\begin{array}{l}\text { Secreted phosphoprotein } 1 \\
\text { (osteopontin, bone sialoprotein I, } \\
\text { early T-lymphocyte activation } 1 \text { ) }\end{array}$ & Extracellular space & NM_009263 & -2.060 \\
\hline TNFRSF9 & $\begin{array}{l}\text { Tumor necrosis factor receptor } \\
\text { superfamily, member } 9\end{array}$ & Plasma membrane & AK019885 & -2.000 \\
\hline TNFRSF11B & $\begin{array}{l}\text { Tumor necrosis factor receptor superfamily, } \\
\text { member } 11 \mathrm{~b} \text { (osteoprotegerin) }\end{array}$ & Plasma membrane & NM_008764 & -2.538 \\
\hline$U B A 1$ & Ubiquitin-like modifier activating enzyme 1 & Cytoplasm & NM_009457 & -2.660 \\
\hline$V T N$ & Vitronectin & Extracellular space & NM_011707 & 2.070 \\
\hline
\end{tabular}

Table IV. Gene expression profiles in MSCs co-cultured with $\mathrm{CCl}_{4}$-injured liver cells.

\begin{tabular}{|c|c|c|c|c|}
\hline Symbol & Name & Location & Accession & Fold Change \\
\hline ADAMTS1 & $\begin{array}{l}\text { ADAM metallopeptidase with } \\
\text { thrombospondin type } 1 \text { motif, } 1\end{array}$ & Extracellular space & NM_009621 & -3.724 \\
\hline ADAMTS5 & $\begin{array}{l}\text { ADAM metallopeptidase with thrombospondin } \\
\text { type } 1 \text { motif, } 5 \text { (aggrecanase-2) }\end{array}$ & Extracellular space & AK046558 & -4.255 \\
\hline$A H R$ & Aryl hydrocarbon receptor & Nucleus & NM_013464 & -2.930 \\
\hline$A K T 1$ & v-akt murine thymoma viral oncogene homolog 1 & Cytoplasm & NM_009652 & -2.083 \\
\hline$A P A F 1$ & Apoptotic peptidase activating factor 1 & Cytoplasm & NM_009684 & -2.209 \\
\hline$B C L 10$ & B-cell CLL/lymphoma 10 & Cytoplasm & AK0̈08820 & -2.532 \\
\hline C3 & Complement component 3 & Extracellular space & NM_009778 & -4.415 \\
\hline CASP3 & Caspase 3 , apoptosis-related cysteine peptidase & Cytoplasm & NM_009810 & -2.597 \\
\hline CASP4 & Caspase 4 , apoptosis-related cysteine peptidase & Cytoplasm & NM_007609 & -2.360 \\
\hline CAVI & Caveolin 1 , caveolae protein, $22 \mathrm{kDa}$ & Plasma membrane & NM_007616 & -2.297 \\
\hline CCL24 & Chemokine (C-C motif) ligand 24 & Extracellular space & NM_019577 & 2.368 \\
\hline CCRL1 & Chemokine (C-C motif) receptor-like 1 & Plasma membrane & NM_145700 & -2.060 \\
\hline CD5 & CD5 molecule & Plasma membrane & NM_007650 & 2.327 \\
\hline$C D 34$ & CD34 molecule & Plasma membrane & NM_133654 & 2.561 \\
\hline CD86 & CD86 molecule & Plasma membrane & NM_019388 & -2.558 \\
\hline
\end{tabular}


Table IV. Continued.

\begin{tabular}{|c|c|c|c|c|}
\hline Symbol & Name & Location & Accession & Fold Change \\
\hline$C D K 2$ & Cyclin-dependent kinase 2 & Nucleus & NM_183417 & -2.091 \\
\hline CFLAR & CASP8 and FADD-like apoptosis regulator & Cytoplasm & NM_207653 & -2.498 \\
\hline COLI8AI & Collagen, type XVIII, $\alpha 1$ & Extracellular space & NM_009929 & -2.239 \\
\hline COL27Al & Collagen, type XXVII, $\alpha 1$ & Extracellular space & AK003879 & 2.572 \\
\hline CUL1 & Cullin 1 & Nucleus & NM_012042 & -2.727 \\
\hline CXCL2 & Chemokine (C-X-C motif) ligand 2 & Extracellular space & NM_008176 & 2.600 \\
\hline CXCL10 & Chemokine (C-X-C motif) ligand 10 & Extracellular space & NM_021274 & -3.448 \\
\hline EFNA5 & Ephrin-A5 & Plasma membrane & NM_010109 & 2.040 \\
\hline EGFR & $\begin{array}{l}\text { Epidermal growth factor receptor } \\
\text { [erythroblastic leukemia viral (v-erb-b) } \\
\text { oncogene homolog, avian] }\end{array}$ & Plasma membrane & NM_207655 & 2.230 \\
\hline$E R B B 2 I P$ & erbb2 interacting protein & Extracellular space & NM_001005868 & -2.089 \\
\hline GNA13 & $\begin{array}{l}\text { Guanine nucleotide binding protein } \\
\text { (G protein), } \alpha 13\end{array}$ & Plasma membrane & NM_010303 & -2.083 \\
\hline GPX1 & Glutathione peroxidase 1 & Cytoplasm & NM_008160 & 2.063 \\
\hline$H G F$ & $\begin{array}{l}\text { Hepatocyte growth factor } \\
\text { (hepapoietin A; scatter factor) }\end{array}$ & Extracellular space & NM_010427 & -2.222 \\
\hline$H N F 4 A$ & Hepatocyte nuclear factor $4, \alpha$ & Nucleus & NM_008261 & 2.144 \\
\hline IDI & $\begin{array}{l}\text { Inhibitor of DNA binding 1, dominant negative } \\
\text { helix-loop-helix protein }\end{array}$ & Nucleus & NM_010495 & 2.113 \\
\hline ID2 & $\begin{array}{l}\text { Inhibitor of DNA binding 2, dominant } \\
\text { negative helix-loop-helix protein }\end{array}$ & Nucleus & NM_010496 & 2.095 \\
\hline$I G F 2$ & Insulin-like growth factor 2 (somatomedin $\mathrm{A})$ & Extracellular space & NM_010514 & -2.547 \\
\hline IGFBP6 & Insulin-like growth factor binding protein 6 & Extracellular space & NM_008344 & 3.185 \\
\hline$I L 1 B$ & Interleukin $1, \beta$ & Extracellular space & NM_008361 & -2.457 \\
\hline ITGA6 & Integrin, $\alpha 6$ & Plasma membrane & AK045391 & -2.360 \\
\hline $\begin{array}{l}\text { KITLG } \\
\text { (includes EG: }\end{array}$ & $\begin{array}{l}\text { KIT ligand } \\
\text { 254) }\end{array}$ & Extracellular space & NM_013598 & -2.876 \\
\hline LAMA3 & Laminin, $\alpha 3$ & Extracellular space & XM_140451 & 2.272 \\
\hline$M A F$ & $\begin{array}{l}\text { v-maf musculoaponeurotic fibrosarcoma } \\
\text { oncogene homolog (avian) }\end{array}$ & Nucleus & NM_001025577 & -2.254 \\
\hline$M A P 2 K 7$ & Mitogen-activated protein kinase kinase 7 & Cytoplasm & ВC070467 & 2.019 \\
\hline$M A P 3 K 1$ & Mitogen-activated protein kinase kinase kinase 1 & Cytoplasm & NM_011945 & -2.091 \\
\hline MCL1 & Myeloid cell leukemia sequence 1 (BCL2-related) & Cytoplasm & NM_008562 & -2.155 \\
\hline NCOR1 & Nuclear receptor co-repressor 1 & Nucleus & AK035813 & -3.040 \\
\hline NFATC2 & $\begin{array}{l}\text { Nuclear factor of activated T-cells, } \\
\text { cytoplasmic, calcineurin-dependent } 2\end{array}$ & Nucleus & AK081853 & -3.096 \\
\hline NGFR & $\begin{array}{l}\text { Nerve growth factor receptor } \\
\text { (TNFR superfamily, member 16) }\end{array}$ & Plasma membrane & NM_033217 & 3.110 \\
\hline NOTCHI & $\begin{array}{l}\text { Notch homolog } 1 \text {, translocation-associated } \\
\text { (Drosophila) }\end{array}$ & Plasma membrane & NM_008714 & -2.865 \\
\hline NRIDI & Nuclear receptor subfamily 1 , group D, member 1 & Nucleus & NM_145434 & -2.275 \\
\hline PKD1 & Polycystic kidney disease 1 (autosomal dominant) & Plasma membrane & NM_013630 & 2.086 \\
\hline PTEN & $\begin{array}{l}\text { Phosphatase and tensin homolog } \\
\text { (mutated in multiple advanced cancers } 1 \text { ) }\end{array}$ & Cytoplasm & AK030750 & -2.000 \\
\hline PTPRC & Pprotein tyrosine phosphatase, receptor type, C & Plasma membrane & NM_011210 & -3.293 \\
\hline$R A R B$ & Retinoic acid receptor, $\beta$ & Nucleus & NM_011243 & 4.876 \\
\hline$R A R G$ & Retinoic acid receptor, $\gamma$ & Nucleus & NM_011244 & 2.050 \\
\hline$R G S 3$ & Regulator of G-protein signaling 3 & Nucleus & NM_134257 & 2.091 \\
\hline SLIT2 & Slit homolog 2 (Drosophila) & Extracellular space & AK038807 & 3.000 \\
\hline STAT1 & $\begin{array}{l}\text { Signal transducer and activator of } \\
\text { transcription } 1,91 \mathrm{kDa}\end{array}$ & Nucleus & AK041814 & -3.25 \\
\hline$T G F B R 3$ & Transforming growth factor, $ß$ receptor III & Plasma membrane & NM_011578 & 2.000 \\
\hline$T H R B$ & $\begin{array}{l}\text { Thyroid hormone receptor, } \beta \text { [erythroblastic } \\
\text { leukemiaviral (v-erb-a) oncogene homolog } 2 \text {, avian] }\end{array}$ & Nucleus & NM_009380 & 2.947 \\
\hline TNFRSF 11B & $\begin{array}{l}\text { Tumor necrosis factor receptor superfamily, } \\
\text { member } 11 \mathrm{~b} \text { (osteoprotegerin) }\end{array}$ & Plasma membrane & NM_008764 & -3.978 \\
\hline
\end{tabular}




\section{Discussion}

In this study, we focused on the therapeutic potential of MSCs in injured liver tissues. Using a microarray containing 44,000 genes, we assessed the gene expression profiles of MSCs in the presence of injured liver cells and normal liver cells. The results demonstrate that MSC gene responses to co-culturing with liver cells occurred in a condition-specific manner.

The results of the microarray analysis of MSCs co-cultured with normal liver cells demonstrate that genes associated with the inflammatory process were upregulated; e.g., CXCR6 (receptor for CXCL16), CCR3 (receptor for RANTES, MCP2, -3, -4), IL-2, IL-11, CD34 and CD74 (Ii chain of class II MHC molecules). Although CXCR6 (5), IL-11 (6) and CD34 (7) were reportedly expressed in human MSCs, these genes were also upregulated in mice MSCs co-cultured with normal liver cells. It is also worth noting that the expression of CD74, an invariant chain of class II MHC molecules, was upregulated. CD74 is required for the macrophage migration inhibitory factor-induced activation of the extracellular signalregulated kinase-1/2 MAP kinase cascade, cell proliferation and $\mathrm{PGE}_{2}$ production (8). The procollagen (type 1 and $\alpha 1$ ) gene was also upregulated and is believed to be associated with the tissue repair process. Neuregulin 4 is one of the neuregulins, a diverse family of EGF-like ligands that are sensitive to ADAM (a disintegrin and metalloproteinase) for the cleavage of the extracellular domain (9). Wnt signaling affects the developmental process of stem cells, including MSCs. In MSCs, signaling via the Wnt/ß-catenin pathway stimulates osteoblastogenesis and inhibits adipogenesis by regulating the relative levels of tissue-specific transcription factors (10). It has also been reported that the Wnt/B-catenin pathway contributes to the activation of liver progenitor cells (11). Therefore, in MSCs co-cultured with normal liver cells, Wnt 2 and catenin were also upregulated and may be associated with differentiation into hepatocytes.

The results of the microarray analysis of MSCs cocultured with liver cells from $\mathrm{CCl}_{4}$-injected mice demonstrate that genes associated with hypoxia response were upregulated; e.g., cytoglobin, hypoxia inducible factor 3 ( $\alpha$ subunit) and erythropoietin. Cytoglobin, the gene involved in cell proliferation - possibly via collagen synthesis - and expressed predominantly in fibroblasts and associated cell types, is significantly elevated under hypoxic conditions (12). In this study, cytoglobin was upregulated in both the liver and $\mathrm{CCl}_{4}$ liver groups. Hypoxia also induces the upregulation of erythropoietin, Sox6, and particularly Sox9, which is a key regulator of the differentiation of MSCs into chondrocytes. Another upregulated gene in the $\mathrm{CCl}_{4}$ liver group was Vav2, the guanine nucleotide exchange factor. The primary function of this gene is the regulation of collagen phagocytosis, which is an $\alpha 2 \beta 1$ integrin-dependent extracellular remodeling process (13). CXCL2 (Groß) is a ligand of CXCR2 and contributes to the rapid mobilization of HSCs with enhanced engraftment properties (14). Upregulation of the v-Erb gene can be considered in the context of epidermal growth factor receptors. In particular, it is noticeable that the expression of hepatic nuclear factor $4 \alpha$, a critical transcription factor in hepatocyte differentiation (15), was upregulated.
MSCs co-cultured with liver cells from $\mathrm{CCl}_{4}$-injected mice evidenced elevated levels of several growth factors, including epidermal growth factors. The observed up-regulation of frizzled homolog 4 reflects the possibility that the Wnt/ßcatenin pathway is relevant to the co-culturing of MSCs with liver cells.

Several genes were upregulated in common in the liver and $\mathrm{CCl}_{4}$ liver groups; e.g., angiotensin receptor-like 1, CD34, procollagen type XXVII, cytochrome P450, fibroblast growth factor, forkhead box G1, hepatocyte nuclear factor 4, inhibitor of DNA binding 1, IL-1, matrix metallopeptidases, retinoic acid receptor $\beta, S 100$ protein $\beta$, slit homologue 2 and tyrosine kinase 2. As well, several genes were downregulated in both groups; e.g., C3, CCR2, ADAM metallopepidase with thrombospondin type 1 and 5, CD86, cullin 1, Erbb2 interacting protein, GNA13, hepatocyte growth factor, integrin $\alpha 6$, NFATc2 and protein tyrosine phosphatase receptor type C. In the liver group, Socs3 was downregulated. Socs3 is a key inhibitor of cytokines that utilize gp130 (e.g., IL-23R and IL-6R), whereas Socs1 is believed to inhibit any cytokines that utilize $\gamma \mathrm{c}$ (16). In the $\mathrm{CCl}_{4}$ liver group, PTEN was downregulated compared to the untreated MSCs. PTEN is a tumor suppressor gene and functions as a lipid phosphatase that decreases the PI3K signaling pathway. In the absence of PTEN, HSCs are driven into the cell cycle; the loss of PTEN frequently promotes the formation of a variety of tumors (17).

In summary, MSCs co-cultured with normal liver cells exhibited the potential for differentiation into functional liver cells via upregulation of the genes associated with inflammatory response. MSCs co-cultured with $\mathrm{CCl}_{4}$ liver cells differentiated into functional liver cells and upregulated genes related to hypoxic stress. In the $\mathrm{CCl}_{4}$-injected mice, hypoxicinduced responses were involved in the regeneration process of liver cells via MSCs. We suggest that a diverse repairing pathway contributes to the regeneration process of liver cells by MSCs.

\section{Acknowledgements}

This work was supported by a Korea Research Foundation Grant funded by the Korean government (KOEHRD, Basic Research Promotion Fund) (KRF-2007-531-E00061).

\section{References}

1. Lange C, Bruns H, Kluth D, Zander AR and Fiegel HC: Hepatocytic differentiation of mesenchymal stem cells in cocultures with fetal liver cells. World J Gastroenterol 12: 2394-2397, 2006.

2. Ong SY, Dai H and Leong KW: Hepatic differentiation potential of commercially available human mesenchymal stem cells. Tissue Eng 12: 3477-3485, 2006.

3. Talens-Visconti R, Bonora A, Jover R, Mirabet V, Carbonell F, Castell JV and Gomez-Lechon MJ: Hepatogenic differentiation of human mesenchymal stem cells from adipose tissue in comparison with bone marrow mesenchymal stem cells. World J Gastroenterol 12: 5834-5845, 2006.

4. Jung YJ, Ryu KH, Cho SJ, Woo SY, Seoh JY, Chun CH, Yoo K, Moon IH and Han HS: Syngenic bone marrow cells restore hepatic function in carbon tetrachloride-induced mouse liver injury. Stem Cells Dev 15: 687-695, 2006.

5. Honczarenko M, Le Y, Swierkowski M, Ghiran I, Glodek AM and Silberstein LE: Human bone marrow stromal cells express a distinct set of biologically functional chemokine receptors. Stem Cells 24: 1030-1041, 2006. 
6. Majumdar MK, Thiede MA, Mosca JD, Moorman M and Gerson SL: Phenotypic and functional comparison of cultures of marrow-derived mesenchymal stem cells (MSCs) and stromal cells. J Cell Physiol 176: 57-66, 1998.

7. Wagner W, Wein F, Seckinger A, Frankhauser M, Wirkner U, Krause U, Blake J, Schwager C, Eckstein V, Ansorge W and Ho AD: Comparative characteristics of mesenchymal stem cells from human bone marrow, adipose tissue, and umbilical cord blood. Exp Hematol 33: 1402-1416, 2005.

8. Leng L, Metz CN, Fang Y, Xu J, Donnelly S, Baugh J, Delohery T, Chen Y, Mitchell RA and Bucala R: MIF signal transduction initiated by binding to CD74. J Exp Med 197: 1467-476, 2003.

9. Hayes NV and Gullick WJ: The neuregulin family of genes and their multiple splice variants in breast cancer. J Mammary Gland Biol Neoplasia 13: 205-214, 2008.

10. Bennett CN, Longo KA, Wright WS, Suva LJ, Lane TF, Hankenson KD and MacDougald OA: Regulation of osteoblastogenesis and bone mass by Wnt10b. Proc Natl Acad Sci USA 102: 3324-3329, 2005.

11. Yang W, Yan HX, Chen L, Liu Q, He YQ, Yu LX, Zhang SH, Huang DD, Tang L, Kong XN, Chen C, Liu SQ, Wu MC and Wang HY: Wnt/beta-catenin signaling contributes to activation of normal and tumorigenic liver progenitor cells. Cancer Res 68: 4287-4295, 2008.
12. Burmester T, Gerlach F and Hankeln T: Regulation and role of neuroglobin and cytoglobin under hypoxia. Adv Exp Med Biol 618: 169-180, 2007.

13. Arora PD, Marignani P and McCulloch CA: Collagen phagocytosis is regulated by the guanine nucleotide exchange factor Vav2. Am J Physiol Cell Physiol 295: C130-C137, 2008.

14. Pelus LM and Fukuda S: Peripheral blood stem cell mobilization: the CXCR2 ligand GRObeta rapidly mobilizes hematopoietic stem cells with enhanced engraftment properties. Exp Hematol 34: 1010-1020, 2006.

15. Kyrmizi I, Hatzis P, Katrakili N, Tronche F, Gonzalez FJ and Talianidis I: Plasticity and expanding complexity of the hepatic transcription factor network during liver development. Genes Dev 20: 2293-2305, 2006.

16. O'Shea JJ and Murray PJ: Cytokine signaling modules in inflammatory responses. Immunity 28: 477-487, 2008.

17. Rossi DJ and Weissman IL: Pten, tumorigenesis, and stem cell self-renewal. Cell 125: 229-231, 2006. 
